\title{
The feasibility and safety of a simple method for coronary sinus blood sampling during catheter ablation of arrhythmias
}

\author{
Zhenda Zheng ${ }^{1 \#}$, Bingyuan Wu ${ }^{1 \#}$, Qian Chen ${ }^{1}$, Yanting Luo ${ }^{1}$, Xixiang Tang ${ }^{2}$, Jiafu Wang ${ }^{1}$, Xujing Xie ${ }^{1}$, \\ Ruiming Dong ${ }^{1}$, Weiqiang $\mathrm{Li}^{3}$, Jieming $\mathrm{Zhu}^{1}$, Suhua $\mathrm{Li}^{1}$ \\ ${ }^{1}$ Department of Cardiovascular Medicine, the Third Affiliated Hospital, Sun Yat-sen University, Guangzhou, China; ${ }^{2}$ VIP Medical Service Center, \\ the Third Affiliated Hospital, Sun Yat-sen University, Guangzhou, China; ${ }^{3}$ Center for Stem Cell Biology and Tissue Engineering, Key Laboratory \\ for Stem Cells and Tissue Engineering, Ministry of Education, Sun Yat-sen University, Guangzhou, China \\ Contributions: (I) Conception and design: Z Zheng, B Wu, S Li; (II) Administrative support: W Li, J Zhu; (III) Provision of study materials or \\ patients: X Xie, R Dong; (IV) Collection and assembly of data: Q Chen, Y Luo; (V) Data analysis and interpretation: B Wu, X Tang, J Wang; (VI) \\ Manuscript writing: All authors; (VII) Final approval of manuscript: All authors. \\ \#These authors contributed equally to this work. \\ Correspondence to: Suhua Li; Jieming Zhu. Department of Cardiovascular Medicine, the Third Affiliated Hospital, Sun Yat-sen University, Tianhe \\ Road, Guangzhou 510630, China. Email: lisuhua3@mail.sysu.edu.cn; zhujming@mail.sysu.edu.cn; Weiqiang Li. Center for Stem Cell Biology and \\ Tissue Engineering, Key Laboratory for Stem Cells and Tissue Engineering, Ministry of Education, Sun Yat-sen University, Guangzhou, China. \\ Email: liweiq6@mail.sysu.edu.cn.
}

Background: Coronary sinus (CS) blood sampling gives an insight into the localized pathophysiology of heart diseases. However, additional specifically-designed or modified catheters were needed in most previous studies, making the convenience of clinical application unsatisfactory. This study aimed to introduce a simple method for CS blood sampling without using additional catheters during catheter ablation (CA) of arrhythmias, and to investigate its feasibility and safety.

Methods: A total of 119 patients undergoing CA were prospectively enrolled, including 25 with paroxysmal supraventricular tachycardia (PSVT), 30 with premature ventricular complexes (PVC), and 64 with atrial fibrillation (AF). Cannulation and sampling of CS was performed via the femoral vein using a conventional $8 \mathrm{~F} \mathrm{SR0}{ }^{\mathrm{TM}}$ or $8.5 \mathrm{~F} \mathrm{SL}^{\mathrm{TM}}$ introducer sheath (St. Jude Medical) guided by a $6 \mathrm{~F}$ steerable diagnostic catheter (St. Jude Medical). The success rate and any suspicious complications were recorded. Untargeted liquid chromatograph-mass spectrometer (LC-MS)-based metabonomics of CS samples versus peripheral venous samples were also performed.

Results: CS blood samples were successfully collected from 114 patients, with an overall success rate of 95.8\%. Among patients with different arrhythmias, the success rates were similar, with 96.0\% in PSVT, 96.7\% in PVC, and 95.3\% in $\mathrm{AF}(\mathrm{P}=0.223)$. Adverse events occurred in four (3.4\%) patients, including two patients with occasional atrial ectopic beats without causing any discomfort, and two patients with new-onset paroxysmal AF lasting for about $2 \mathrm{~min}$. No serious complications were noted. Metabonomics analysis showed that CS samples provided a number of different metabolites (93 in PVST, 217 in PVC, and 109 in AF) versus peripheral samples.

Conclusions: Our method for CS blood sampling during CA is feasible and safe, and can provide useful cardiometabolic information that is significantly different from a peripheral sample.

Keywords: Coronary sinus (CS); catheter ablation (CA); arrhythmias; atrial fibrillation (AF); metabonomics

Submitted Nov 30, 2021. Accepted for publication Feb 11, 2022.

doi: $10.21037 /$ atm-21-6973

View this article at: https://dx.doi.org/10.21037/atm-21-6973 


\section{Introduction}

Circulating factors can provide a wealth of valuable information regarding the pathophysiology of heart diseases. However, samples from peripheral blood only reflect a systemic rather than a heart-specific level. Therefore, other samples that specifically reflect the local cardiac microenvironment are warranted. Coronary sinus (CS) is a venous channel that is connected to the largest cardiac veins, and receives most of the blood from the walls of the heart. Many cardiac procedures, such as ablation of arrhythmias, left ventricular pacing, retrograde cardioplegia, targeted drug delivery, and stem cell therapy, utilize CS (1), and provide opportunities for CS blood collection. CS samples can reflect heart-specific measurements of myocardial metabolism, offering important clinical information for the diagnosis, treatment, and prognosis of heart diseases $(2,3)$.

Previous studies have shown a good correlation between indices from CS blood sampling and clinical outcomes of heart diseases, including heart failure $(4,5)$, coronary artery disease (6), and Kawasaki disease. However, studies on CS blood sampling in patients with arrhythmia are rare. In addition, specifically-designed or modified catheters for CS blood sampling were additionally needed in most previous studies $(7,8)$, and therefore, the convenience of clinical application is unsatisfactory. In recent years, technologies for intracardiac electrophysiologic study and catheter ablation (CA) for arrhythmias have been developed rapidly, making it possible to obtain CS blood samples using conventional electrophysiological catheters (9). In the present study, we aimed to introduce an easy and convenient method for CS blood sampling during CA for arrhythmias, without using specifically-designed or modified catheters. The feasibility and safety of this method was also assessed.

Metabolomics takes body fluids, cells and tissues as research objects, uses chromatography-mass spectrometry, magnetic resonance technology and other tools to qualitatively or quantitatively analyze endogenous low molecular weight compounds in the body, study abnormal metabolic pathways in the body, and provide scientific diagnosis basis. It has been successfully applied in the early diagnosis of cardiovascular disease, assessment of disease prognosis and treatment effect. In the present study, untargeted liquid chromatograph-mass spectrometer (LCMS) based metabonomics of CS samples versus peripheral venous samples were also performed. We present the following article in accordance with the STROBE reporting checklist (available at https://atm.amegroups.com/article/ view/10.21037/atm-21-6973/rc).

\section{Methods}

\section{Study population}

This is a single-center prospective study conducted in the Third Affiliated Hospital of Sun Yat-sen University between September 2020 and August 2021. Consecutive adult patients with arrhythmias who met guideline indications for CA were enrolled. Predefined arrhythmias included paroxysmal supraventricular tachycardia (PSVT), frequent premature ventricular complexes (PVC), and atrial fibrillation (AF). The diagnosis of PSVT, PVC and AF was based on the patients' medical records and on a 12-channel ECG. ECGs were analysed by skilled physicians. Patients aged $<18$ years old, and those with hematopoietic diseases, malignant tumor, and pregnancy were excluded. The study was conducted in accordance with the Declaration of Helsinki (as revised in 2013). Ethical approval was obtained from the Ethics Committee of the Third Affiliated Hospital of Sun Yat-sen University [No. (2020)02-196-01]. Informed consent was obtained from all participants.

\section{Coronary sinus blood sampling}

Cannulation of the CS was performed via the femoral vein during CA. After puncturing the right femoral vein, a conventional $8 \mathrm{~F} \mathrm{SR} 0^{\mathrm{TM}}$ or $8.5 \mathrm{~F} \mathrm{SL} 1^{\mathrm{TM}}$ introducer sheath (St. Jude Medical, USA), selected by the requirements of CA, was advanced over a guidewire into the lower right atrium and oriented toward the tricuspid valve. The guidewire and the inner sheath were removed, and the outer sheath was flushed with heparinized saline. Next, a $6 \mathrm{~F}$ steerable diagnostic catheter (St. Jude Medical) was inserted into the outer sheath, advanced to the right atrium, flexed with applied torque, and placed stably into the CS using a routine method in a $45^{\circ}$ left anterior oblique projection.

The CS ostium was located by typical electrogram of the proximal CS 9.0 electrode pair. The introducer sheath was then slightly advanced over the diagnostic catheter, engaging the tip into the position of the CS 7.8 electrode pair. The position of the introducer sheath inside the CS was further confirmed and recorded with the injection of contrast medium; otherwise, the process was repeated until successful CS insertion was achieved. Once the position was realized, a $10 \mathrm{~mL}$ CS blood sample was drawn from the introducer sheath. Another $10 \mathrm{ml}$ of peripheral venous sample was collected at the same time. The samples were allowed to clot for $15 \mathrm{~min}$ and centrifuged immediately at 3,300 rpm for $10 \mathrm{~min}$. The samples were then aliquoted and 
stored at $-80{ }^{\circ} \mathrm{C}$ until use. After sampling, the introducer sheath was gently removed from the CS and withdrawn into the lower right atrium, preparing for the further CA operation.

The feasibility of CS blood sampling was assessed by calculating the success rate of CS cannulation, which was defined by the achievement of a stable introducer sheath position in a $45^{\circ}$ left anterior oblique projection confirmed by CS angiogram, without causing patient discomfort. The safety of CS blood sampling was evaluated by recording any suspicious complications, including atrioventricular block, unexpected arrhythmias, atrial trauma, pericardial effusion, cardiac perforation, and hematoma or arteriovenous fistula of the femoral vein.

\section{Metabonomics analysis}

Untargeted LC-MS-based metabonomics analysis was performed in 35 patients (19 with AF, eight with PSVT, and eight with PVC), who were matched by age, gender, and risk factors. The high-performance liquid chromatography tandem high-resolution mass spectrometer Q-Exactive (Thermo, Germany) was used to perform metabolome detection of serum samples in positive and negative ion modes, combined with biological information analysis for mass spectrometry data interpretation. Bioinformatics analysis mainly employed XCMS software (Bioconductor) for substance detection, and metaX software for substance quantification and differential substance screening. MetaX software was also used to annotate the first-level mass spectrum of the substance, and the in-house library was used to annotate the metabolites of the second-level mass spectrum of the substance. Multiples and statistical tests for each comparison group were evaluated using a volcano map, and then a heat map was used to show the different substances screened by statistical methods.

\section{Statistical analysis}

Statistical analysis was performed by using SPSS 22.0 for Windows (SPPS Inc., Chicago, IL, USA). Descriptive statistics were presented as means \pm standard deviation for continuous variables, or as numbers and percentages for categorical variables. Differences between continuous variables were evaluated by the student's $t$-test or analysis of variance, as appropriate. Differences between categorical variables were evaluated by the Pearson Chi-square test.
Both the success rate and adverse event rate were calculated in all patients and compared among patients with different arrhythmias.

The main purpose of metabolomics analysis was to screen out metabolites with statistical and biological significance from a large number of detected metabolites. Using the open source MetaX metabolomics analysis process, univariate and multivariate analysis can be performed to obtain differential metabolites between groups, using methods including parametric and nonparametric tests, differential expression multiple analysis, principal component analysis (PCA), partial least squares multiplicative discriminant analysis PLS-DA ( $>3$ samples), and so on. In all of the analyses, a two-tailed value of $\mathrm{P}<0.05$ was considered to indicate statistical significance.

\section{Results}

A total of 119 consecutive patients ( 75 male and 44 female; mean age: 55.7 years) who planned to undergo CA were enrolled within 1 year, including 25 with PSVT, 30 with PVC, and 64 with AF. Compared with the other two groups, patients in the AF group were older on average and had a higher proportion of hypertension and diabetes. There were no significant differences between the three groups in terms of gender, body mass index (BMI), and smoking. Regarding laboratory test indicators, patients in the AF group had lower low-density lipoprotein cholesterol (LDL-C) levels and longer prothrombin time, which is considered to be related to medication. In addition, compared with the other two groups of patients, the proportion of patients in the AF group taking hypoglycemic drugs, lipid-lowering drugs, and anticoagulants was significantly higher, while the proportion of patients taking antiplatelets was lower. The patients' demographics and baseline characteristics are summarized in Table 1.

\section{Feasibility and safety of CS blood sampling}

The steerable diagnostic catheter was successfully placed into the CS in all participants. In 114 of the 119 included patients, the introducer sheath was successfully engaged into the CS with a stable position and CS blood samples were collected, with a success rate of $95.8 \%$ (Figures 1,2; Table 2). The success rates of blood sampling were similar among patients with different arrhythmias $(\mathrm{P}=0.223)$. The reasons for failed cannulation included difficult advancing of 
Table 1 Baseline characteristics of study participants

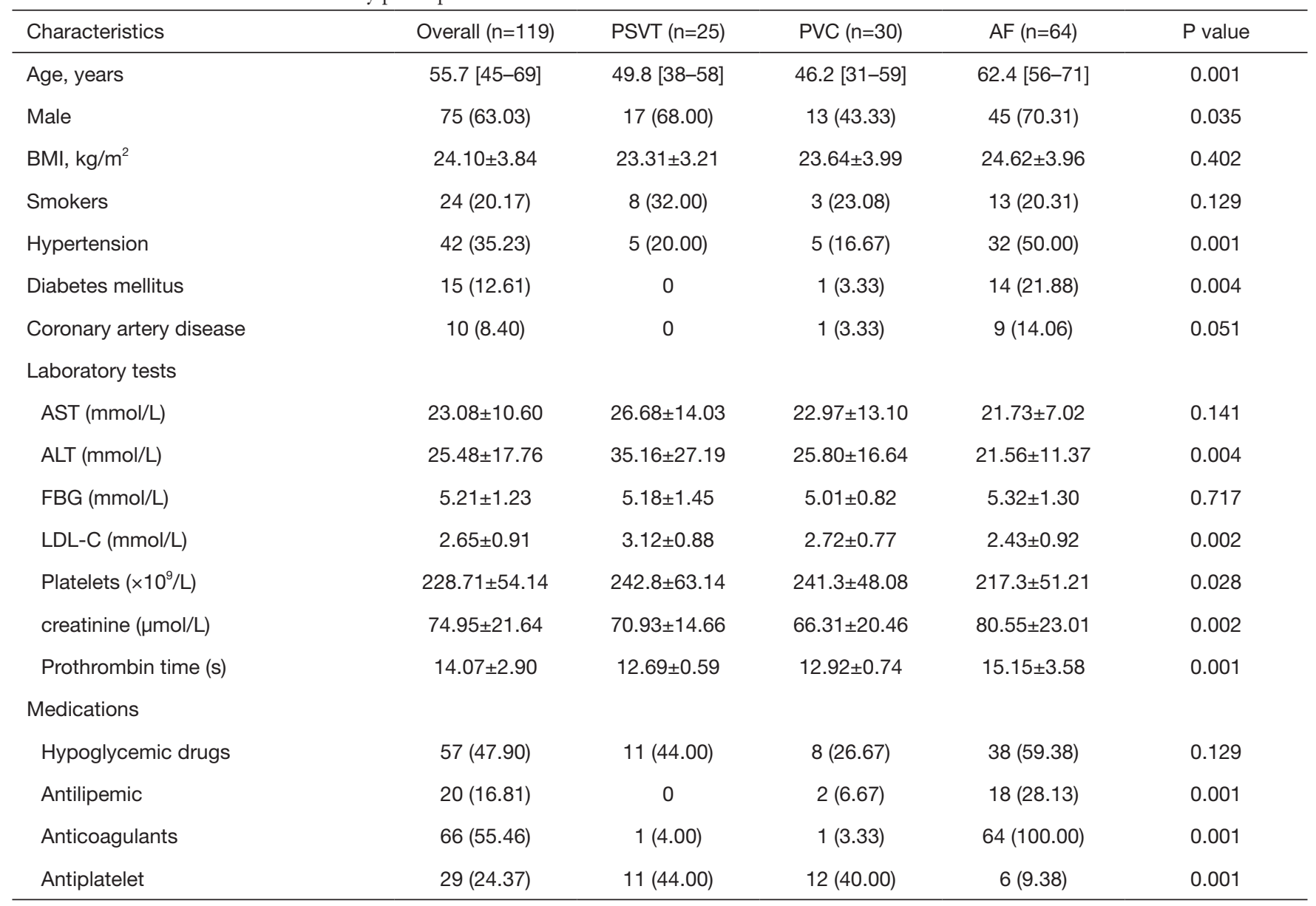

Data are presented as mean (SD), median $\left[25^{\text {th }}\right.$ to $75^{\text {th }}$ percentile] or $\mathrm{n}(\%)$. AF, atrial fibrillation; PSVT, paroxysmal supraventricular tachycardia; PVC, premature ventricular complexes; BMI, body mass index; AST, aspartate aminotransferase; ALT, alanine aminotransferase; FBG, fasting blood glucose; LDL-C, low-density lipoprotein cholesterol.

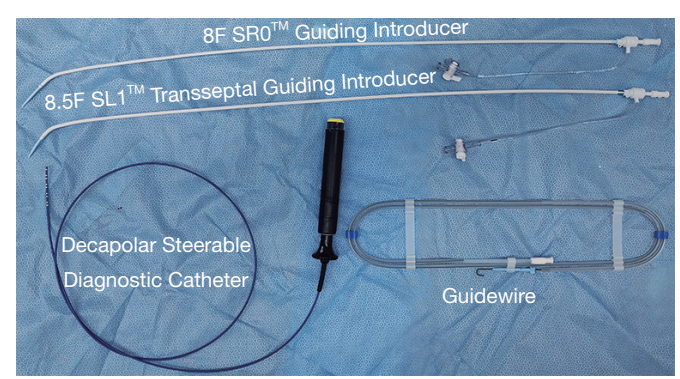

Figure 1 Guidewire and catheters used in CS cannulation. CS, coronary sinus.

introducer sheath to the CS ostium ( $\mathrm{n}=2$, Figure 3), unstable sheath position $(\mathrm{n}=1)$, and patient discomfort $(\mathrm{n}=2)$.

Adverse events occurred in 4 (3.4\%) patients (Table 2).
Occasional atrial ectopic beats were noted in two patients, without causing any discomfort. Paroxysmal AF occurred in two patients without previous history of AF, lasting for about $2 \mathrm{~min}$ and then spontaneously restoring sinus rhythm. No serious complications were noted. The adverse event rates were similar among patients with different arrhythmias $(\mathrm{P}=0.844)$.

\section{Metabonomics analysis}

Metabonomic analysis of CS samples versus peripheral samples found various kinds of differential metabolites in patients with different arrhythmias. In general, there were 217 kinds of differential metabolites in PVST patients, 93 in PVC patients, and 109 in AF patients (Figure 4). 

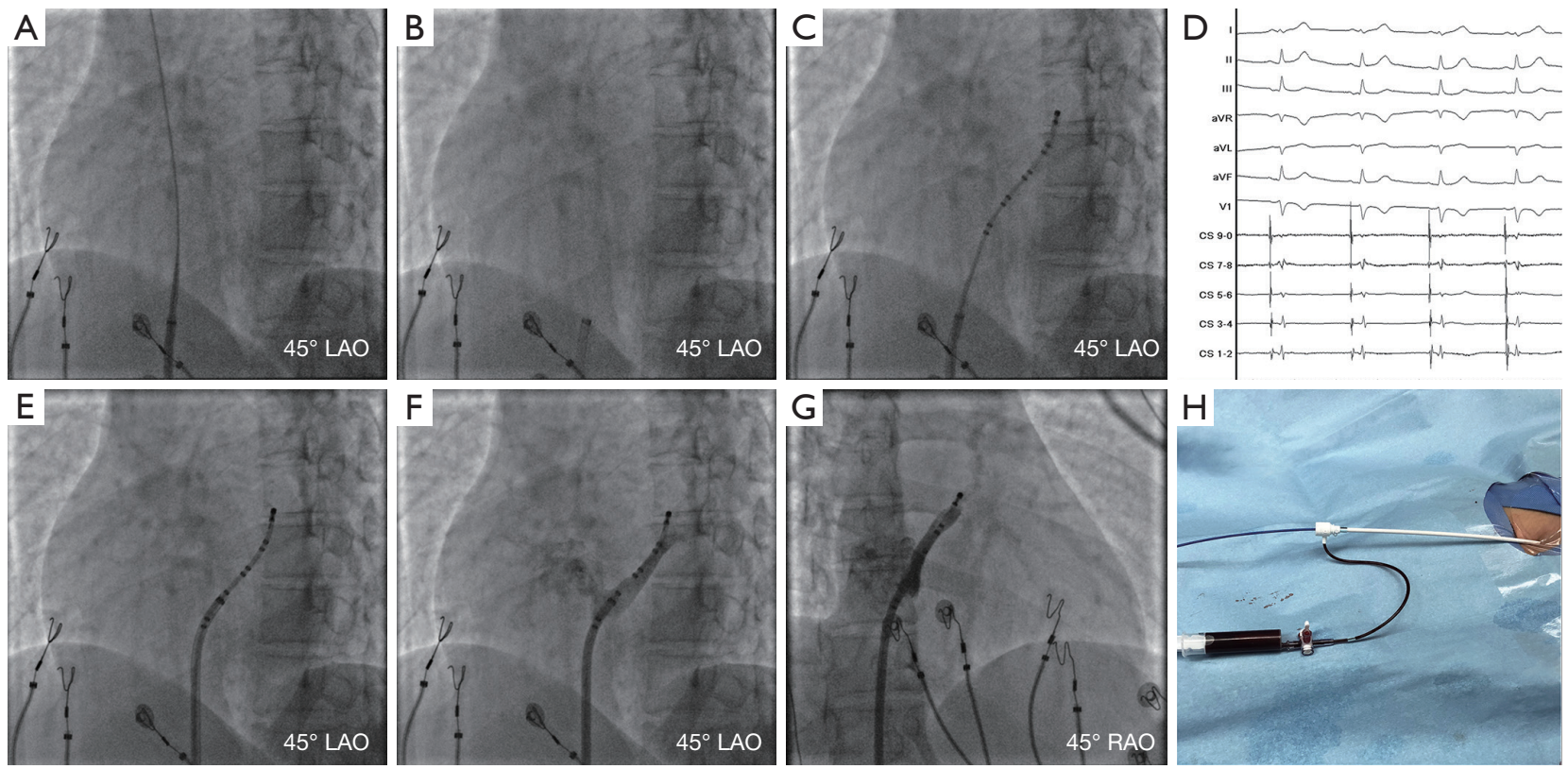

Figure 2 Cannulation of CS via the femoral vein in a 25 -year-old female with frequent premature ventricular complexes. (A) An $8 \mathrm{~F}$ SR0 ${ }^{\mathrm{TM}}$ introducer sheath was advanced over a guidewire into the lower right atrium. (B) The guidewire and the inner sheath were removed. (C) A 6F steerable diagnostic catheter was inserted into the outer sheath and placed into CS. (D) The CS ostium was located by typical electrogram of CS 9-0 electrode pair. (E) The introducer sheath was advanced over the diagnostic catheter, engaging the tip into the position of CS 7-8 electrode pair. (F,G) The position of the introducer sheath was confirmed by CS angiography. (H) Ten mL CS blood sample was drawn from introducer sheath. CS, coronary sinus.

Table 2 Feasibility and safety of CS blood sampling

\begin{tabular}{lccccc}
\hline CS sampling & Overall $(\mathrm{n}=119)$ & PSVT $(\mathrm{n}=25)$ & PVC $(\mathrm{n}=30)$ & AF $(\mathrm{n}=64)$ & $\mathrm{P}$ value \\
\hline Success, $\mathrm{n}(\%)$ & $114(95.8)$ & $24(96.0)$ & $29(96.7)$ & $61(95.3)$ & 0.223 \\
Adverse events, $\mathrm{n}(\%)$ & $4(3.4)$ & $1(4.0)$ & $1(3.3)$ & $2(3.1)$ & 0.844 \\
\hline
\end{tabular}

CS, coronary sinus; PSVT, paroxysmal supraventricular tachycardia, PVC, premature ventricular complexes; AF, atrial fibrillation.
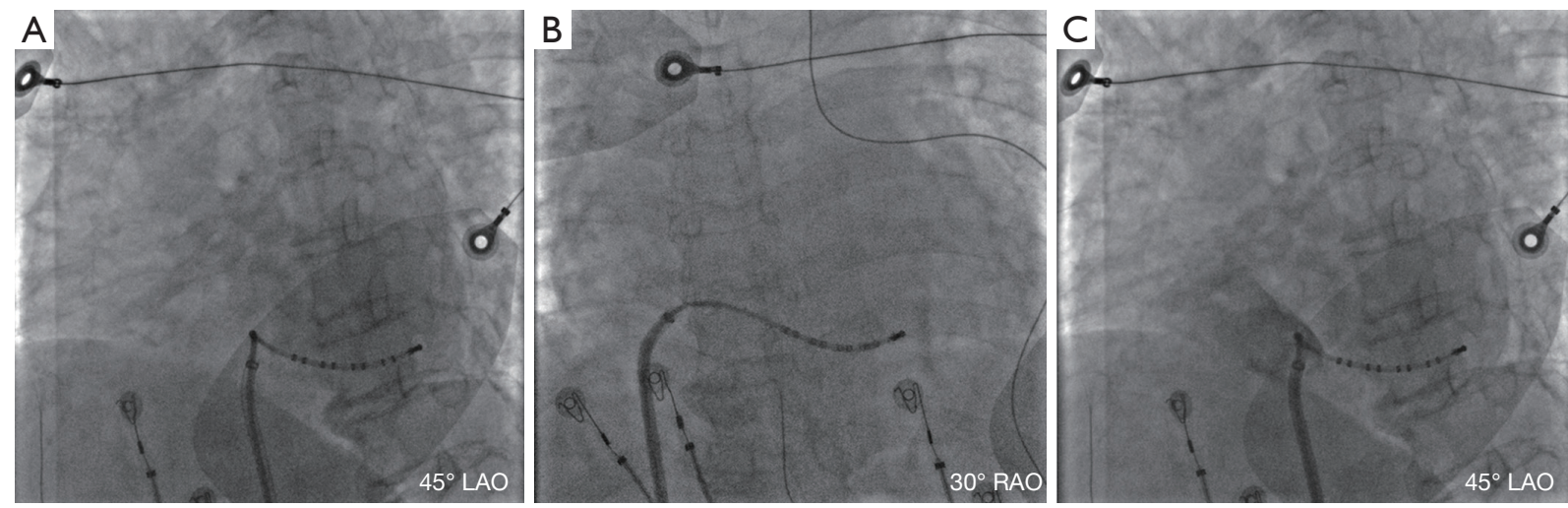

Figure 3 Failed cannulation of CS in a 71-year-old female with persistent atrial fibrillation. (A,B) The introducer sheath was difficult to be advanced to the CS ostium over the diagnostic catheter due to the narrow angle between the CS and the right atrium; (C) angiography showed that the tip of the introducer sheath was not placed in the CS. CS, coronary sinus. 

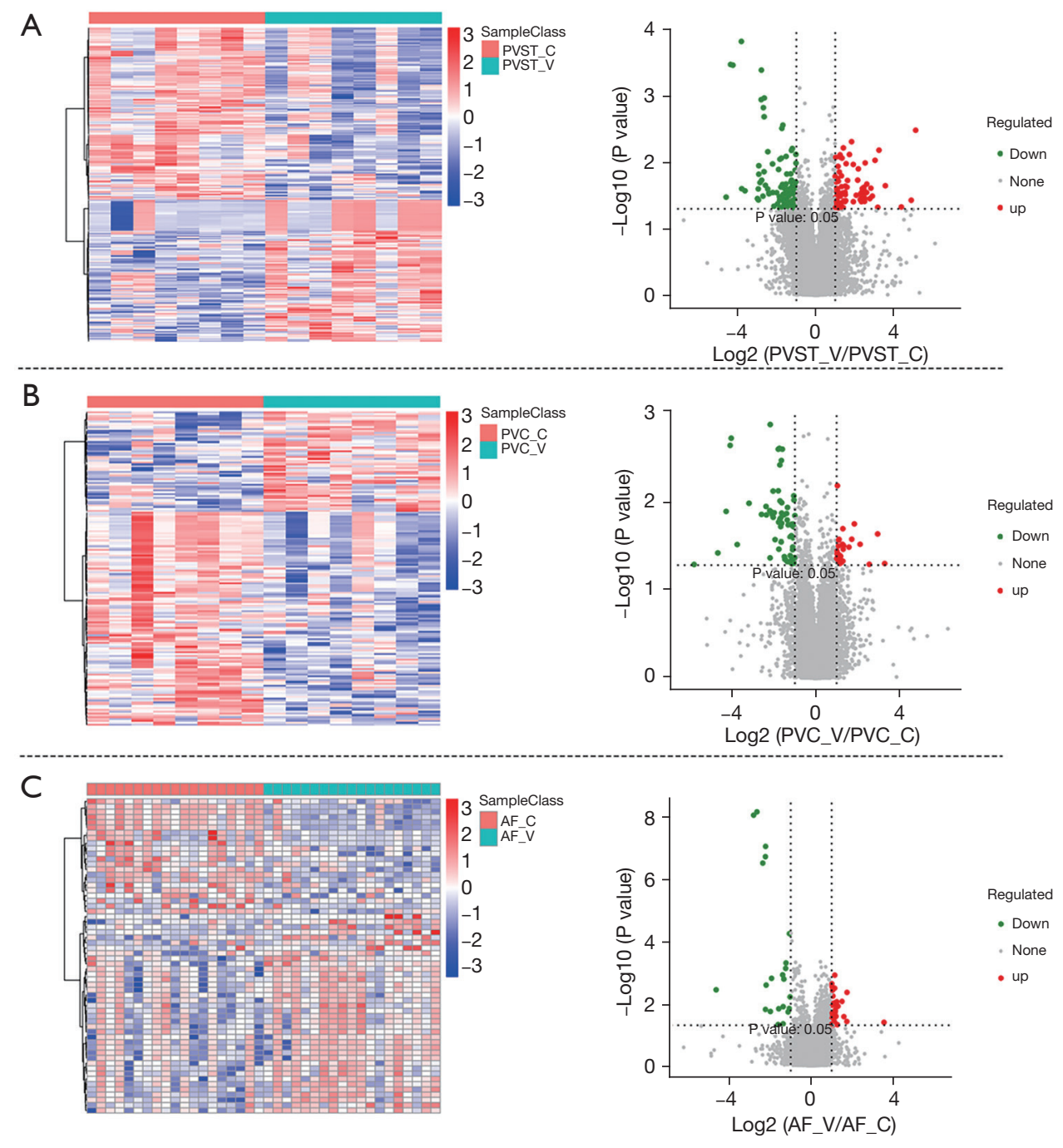

Figure 4 Metabonomics analysis. Univariate statistical tests were demonstrated using a volcano map. The abscissa is the mean intensity ratio of the two phenotypes, and the ordinate is the p-value of statistical test, and the $-\log 10$ was taken. A heat map was used to show each peak of the differential matter. Each row is a decaying peak, and each column is a sample. (A) Differential metabolites between CS and peripheral blood in PVST patients; (B) differential metabolites between CS and peripheral blood in PVC patients; (C) differential metabolites between CS and peripheral blood in AF patients. CS, coronary sinus; PVC, premature ventricular complexes; AF, atrial fibrillation.

\section{Discussion}

The detection of heart-specific biomarkers can help us understand the pathogenesis of many cardiovascular diseases, and guide the treatment of related diseases and the analysis of prognosis (3). Although these biomarkers can be measured in peripheral blood, they lack sufficient sensitivity and specificity due to dilution in the entire systemic circulation (10). Sampling from the CS is a more accurate reflection of cardiometabolic conditions, but it is more invasive, time-consuming, and technically difficult than peripheral sampling; thus, it has not been widely implemented.

CS sampling using a $6.5 \mathrm{~F}$ Simmons II catheter was described in 1986 (11). Ten years later, Winters et al. published a comparative study using multipurpose, Simmons I or II or a modified Simmons catheters through the right femoral vein (12). In 2014, Martínez et al. (8) modified the technique described by Dehmer et al. (11) using a $5 \mathrm{~F}$ right femoral vein sheath and $5 \mathrm{~F}$ Simmons I (for females) and II (for males) catheters. Overall, they reported 
a $99 \%$ success rate in 82 cases, and $2.43 \%$ rate of minor complications, as well as two cases of non-sustained atrial arrhythmias whilst manipulating the catheter in the right atrium (8). The above-mentioned studies all required the use of specially-designed catheters for blood collection. The innovation of this study is that the sheath tube that should be used for catheter ablation is used for blood collection. This method is simple and convenient, and does not increase the economic burden. At the same time, this study used a steerable catheter to guide, locate, and stabilize the position of the sheath inside the CS, which is a less reported method in the literature.

In previous studies, CS intubation is relatively safe, and the main complications include transient supraventricular arrhythmias and catheter position instability. In addition, activation of platelets and thrombin production remains a potential drawback of CS intubation and operation, although this effect can be attenuated with the use of heparin-bound catheters. In this study, a conventional radiofrequency ablation catheter was used for CS blood collection, and the position of the catheter was confirmed by CS angiography. The operation was simple, the success rate was high, and no serious complications occurred, and thus, should encourage research groups to assay CS blood when studying cardiac biomarkers. This simple method of blood collection was initiated by our center and has been widely used in our center. We plan to popularize it to other hospitals.

Metabolomics is considered a tool for the study of biomarkers, mainly used to describe metabolites in biological fluids, cells and tissues (13). Metabolomics based on untargeted and targeted mass spectrometry is the main method for metabolite recovery and identification (14). Metabolomics research methods are mainly based on gas chromatography or liquid chromatography mass spectrometry (GC-MS or LC-MS), which reveal changes in metabolic pathways including glycolysis, fatty acid oxidation, and lipid biosynthesis.

At present, metabonomics is widely used in the study of cardiovascular diseases such as myocardial infarction, heart failure, and arrhythmia. A prospective cohort study in 2017 found that the concentration of arginine in the serum samples of patients with myocardial infarction increased, and the decrease of lysophosphatidylcholine (LPC) 17:0 and LPC 18:2 increased the risk of myocardial infarction (MI) which can be used as a potential biomarker for the diagnosis of MI (15). Murashige et al. used metabolomics on artery, CS, and femoral vein blood in 110 patients with or without heart failure to quantify the uptake and release of 277 metabolites, including all major nutrients, by the human heart and leg. They found that the failing heart consumed more ketones and lactate, and had higher rates of proteolysis. These data provide a comprehensive and quantitative picture of human cardiac fuel use (2). However, CS serum metabolomics analysis of patients with arrhythmia has not been reported in previous literatures. We found that there were 217 kinds of differential metabolites in PVST patients, 93 in PVC patients, and 109 in AF patients. In future research, we will further investigate these differential metabolites, which can provide a potential target for followup diagnosis, treatment, and prognosis research.

\section{Limitations}

There were some cases of failure in this study, mainly in patients with small angle of CS sinus. For these patients, an improved sheath tube with more curved sheath head can be considered.

\section{Acknowledgments}

Funding: This work was supported by the National Science Foundation of China (81900320, 82000278); the Guangdong Basic and Applied Basic Research Foundation (2020A1515010599); the Guangdong Medical Research Foundation (C2019107, A2020142); the Guangzhou Science and Technology Project (202102080388).

\section{Footnote}

Reporting Checklist: The authors have completed the STROBE reporting checklist. Available at https://atm. amegroups.com/article/view/10.21037/atm-21-6973/rc

Data Sharing Statement: Available at https://atm.amegroups. com/article/view/10.21037/atm-21-6973/dss

Conflicts of Interest: All authors have completed the ICMJE uniform disclosure form (available at https://atm. amegroups.com/article/view/10.21037/atm-21-6973/coif). The authors have no conflicts of interest to declare.

Ethical Statement: The authors are accountable for all aspects of the work in ensuring that questions related to the accuracy or integrity of any part of the work are appropriately investigated and resolved. The study was conducted in accordance with the Declaration of Helsinki 
(as revised in 2013). Ethical approval was obtained from the Ethics Committee of the Third Affiliated Hospital of Sun Yat-sen University [No. (2020)02-196-01]. Informed consent was obtained from all participants.

Open Access Statement: This is an Open Access article distributed in accordance with the Creative Commons Attribution-NonCommercial-NoDerivs 4.0 International License (CC BY-NC-ND 4.0), which permits the noncommercial replication and distribution of the article with the strict proviso that no changes or edits are made and the original work is properly cited (including links to both the formal publication through the relevant DOI and the license). See: https://creativecommons.org/licenses/by-nc-nd/4.0/.

\section{References}

1. Shah SS, Teague SD, Lu JC, et al. Imaging of the coronary sinus: normal anatomy and congenital abnormalities. Radiographics 2012;32:991-1008.

2. Murashige $\mathrm{D}$, Jang $\mathrm{C}$, Neinast $\mathrm{M}$, et al. Comprehensive quantification of fuel use by the failing and nonfailing human heart. Science 2020;370:364-8.

3. Jaumdally R, Varma C, Macfadyen RJ, et al. Coronary sinus blood sampling: an insight into local cardiac pathophysiology and treatment? Eur Heart J 2007;28:929-40.

4. Szczurek-Wasilewicz W, Szyguła-Jurkiewicz B, Skrzypek $\mathrm{M}$, et al. Fetuin-A and sodium concentrations are independently associated with all-cause mortality in patients awaiting heart transplantation. Pol Arch Intern Med 2021;131:16081.

5. Ajijola OA, Chatterjee NA, Gonzales MJ, et al. Coronary Sinus Neuropeptide Y Levels and Adverse Outcomes in Patients With Stable Chronic Heart Failure. JAMA Cardiol 2020;5:318-25.

6. Barraclough JY, Joglekar MV, Januszewski AS, et al. A MicroRNA Signature in Acute Coronary Syndrome Patients and Modulation by Colchicine. J Cardiovasc

Cite this article as: Zheng $Z, W u$ B, Chen Q, Luo Y, Tang X, Wang J, Xie X, Dong R, Li W, Zhu J, Li S. The feasibility and safety of a simple method for coronary sinus blood sampling during catheter ablation of arrhythmias. Ann Transl Med 2022;10(4):170. doi: 10.21037/atm-21-6973
Pharmacol Ther 2020;25:444-55.

7. Selvaraj RJ, Sarin K, Singh VR, et al. Radiofrequency ablation of posteroseptal accessory pathways associated with coronary sinus diverticula. J Interv Card Electrophysiol 2016;47:253-9.

8. Martínez GJ, Bailey BP, Celermajer DS, et al. A safe and easy technique to sample the coronary sinus-facilitating a closer look at cardiac disease. Int J Cardiol 2014;176:1321-2.

9. Voros G, Ector J, Garweg C, et al. Increased Cardiac Uptake of Ketone Bodies and Free Fatty Acids in Human Heart Failure and Hypertrophic Left Ventricular Remodeling. Circ Heart Fail 2018;11:e004953.

10. Maier W, Altwegg LA, Corti R, et al. Inflammatory markers at the site of ruptured plaque in acute myocardial infarction: locally increased interleukin-6 and serum amyloid A but decreased C-reactive protein. Circulation 2005;111:1355-61.

11. Dehmer GJ, Schmitz JM, Malloy CR. A new technique for cannulation of the coronary sinus from the femoral vein. Cathet Cardiovasc Diagn 1986;12:426-9.

12. Winters KJ, Lasala JM, Eisenberg PR, et al. Modified heparin-bonded catheter for cannulation of the coronary sinus from the femoral vein. Cathet Cardiovasc Diagn 1996;39:433-7.

13. Johnson CH, Ivanisevic J, Siuzdak G. Metabolomics: beyond biomarkers and towards mechanisms. Nat Rev Mol Cell Biol 2016;17:451-9.

14. Vinaixa M, Schymanski EL, Neumann S, et al. Mass spectral databases for LC/MS- and GC/MS-based metabolomics: State of the field and future prospects. TrAC Trends in Analytical Chemistry 2016;78:23-35.

15. Ward-Caviness CK, $\mathrm{Xu}$ T, Aspelund T, et al. Improvement of myocardial infarction risk prediction via inflammation-associated metabolite biomarkers. Heart 2017;103:1278-85.

(English Language Editor: A. Kassem) 